\title{
On Commutativity of Semiprime Right Goldie $C_{k}$-Rings
}

\author{
Najat M. Muthana, S. Khalid Nauman \\ Department of Mathematics, King Abdulaziz University, Jeddah, KSA \\ Email: \{najat_muthana, synakhaled\}@hotmail.com
}

Received December 2, 2011; revised February 5, 2012; accepted February 14, 2012

\begin{abstract}
This short exposition is about some commutativity conditions on a semiprime right Goldie $C_{k}$-ring. In particular, it is observed here that a semiprime right Goldie $C_{k}$-ring with symmetric quotient is commutative. The statement holds if the symmetric ring is replaced by reduced, 2-primal, left duo, right duo, abelian, NI, NCI, IFP, or Armendariz ring.
\end{abstract}

Keywords: Semiprime Right Goldie $C_{k}$-Rings; Reduced; Symmetric; Von Neumann Regular Rings

In this short note we expose some commutativity conditions on a semiprime right Goldie $C_{k}$-ring. All rings here are associative with an identity. A ring $A$ is said to be a $C_{k}$-ring, as introduced by Chuang and Lin in 1989 in [1], if for very pair of elements $x, y \in A$, there exist integers $m=m(x, y)$ and $n=n(x, y)$ such that

$$
\left[x^{m}, y^{n}\right]_{k}=0 \text {, }
$$

where $[x, y]_{k}$ is the $k$ th-commutator defined by Klein, Nada, and Bell in [2] in 1980, as

$$
[x, y]_{k}=\left[[x, y]_{k-1}, y\right] \text { where }[x, y]_{1}=[x, y] .
$$

A ring is called a symmetric ring (in the sense of Lambek [3]), if whenever $r a b=0$, then $r b a=0, \forall r, a, b \in A$, semiprime (respt. reduced) if $A$ has no non-zero nilpotent ideal (respt. element) and von Neumann regular if for each $a \in A$, there exists $r \in A$ such that $\operatorname{ara}=a$. A ring is right Goldie in case it has finite right uniform dimension and satisfies acc on right annihilators.

In [1; Theorem 1] Chaung-Lin proved that:

Lemma 1: Every reduced $C_{k}$-ring is commutative.

We use it to prove the following.

Theorem: A semiprime right Goldie $C_{k}$-ring with symmetric right quotient is commutative.

Proof: Lambek in [3; Section 1G] proved that every reduced ring is symmetric. We prove that the converse holds for von Neumann regular rings. In deed, one may deduce easily that $A$ is symmetric if and only if

$$
a_{1} a_{2} \cdots a_{k}=0 \text {, }
$$

then

$$
a_{p(1)} a_{p(2)} \cdots a_{p(k)}=0,
$$

where $a_{i}, a_{p(i)} \in A$ and $p$ is a one-to-one correspondence on the set $\{1,2, \cdots, k\}$. Let $a \in N(A)$ be a non-zero ele- ment of some index $n$. Since $A$ is von Neumann regular, for some $x \in A$,

$$
a=\operatorname{axa}=(a x)^{n-1} a .
$$

But $A$ is symmetric and $a^{n}=0$, which implies that $a=$ $a^{n} x^{n-1}=0$. Hence $A$ is reduced.

The famous Goldie's Theorem states that a ring $A$ is semiprime right Goldie if and only if $A$ has a right quotient ring $B$ which is semisimple Artinian [4; Theorem 2.3.6]. But a semisimple Artinian ring is von Neumann regular [5; Theorem 1.7]. Since $B$ is symmetric and now von Neumann regular, therefore $B$ is reduced. This means that $A$ is reduced. Since $A$ is a $C_{k}$-ring, by the Lemma 1 , we get that $A$ is commutative.

The statement of the Theorem remains unchanged if we replace the condition of the ring being symmetric by 2-primal, abelian, left or right duo, NI, NCI, IFP, quasiIFP, near-IFP, Armendariz, weak-Armendariz, and some other relations that are listed in Lemma 2.

Let us denote by $N(A)$ the set of all nilpotent elements of $A$. For a reduced ring $N(A)=0$, and a ring is $N I$ if $N(A)$ is an ideal [6], $N C I$ if $N(A)$ contains a nonzero ideal [7], and 2-primal if $N(\mathrm{~A})$ is the intersection of prime ideals [8]. A ring $A$ is said to have "Insertion of factor property (in short, IFP) [9] in case for any pair of elements $a, b$ of $A$, if $a b=0$, then $a r b=0$ for all $r \in A$. Such rings are also termed as semicommutative in literature, we simply call them IFP rings. Near-IFP (respt. quasi-IFP) rings are introduced recently in [10] (respt. in [11]), and are characterized as $A a A$ contains a non-zero nilpotent ideal of $A$ for any $0 \neq a \in A$ in [10; Proposition 1.2] (respt. $A a A$ is a nilpotent ideal of $A$ for any $0 \neq a \in A$ in [11; Lemma 1.3]).

By definitions, every reduced ring is an $I F P$ ring, an $I F P$ ring is a quasi- $I F P$ ring, and a quasi-IFP ring is a 
near-IFP ring. The converse need not be true in general (see the Example below) but for a semiprime ring it holds.

A ring $\mathrm{A}$ is called Armendariz in [12] if whenever polynomials in $A[x], f(x)=a_{0}+a_{1} x+\cdots+a_{m} x^{m}$ and $g(x)=b_{0}+b_{1} x+\cdots+b_{n} x^{n}$ satisfy $f(x) g(x)=0$, then for each $i, j, a_{i} b_{j}=0$ and weak Armendariz in [13] if whenever $\left(a_{0}+a_{1} x\right)\left(b_{0}+b_{1} x\right)=0$, then for each $i, j$, $a_{i} b_{j}=0$.

For several interactions and various characterizations with examples and counter examples of these rings which we have discussed above the interested reader may refer to the articles $[4,11,12,14,15]$.

Example: It is clear from the examples and counter examples in above citations that the rings listed above are different from each other, but we found no example for near- $I F P$ and quasi- $I F P$ rings to be different in literature. By definitions, quasi- $I F P$ is near-IFP, we prove that the opposite may not be true.

Let $R$ be a ring and $I \neq 0$ a nilpotent ideal of $R$ such that every element of $R-I$ is a unit. For example, a local ring is of this type. By Proposition 1.10 [10] $R_{n}=\operatorname{Mat}_{n}(R)$ is near-IFP. Let $0 \neq\left(r_{i j}\right) \in N\left(R_{n}\right)$. It is clear that

$$
R_{n}\left(r_{i j}\right) R_{n}=R_{n}
$$

is nilpotent if $r_{i j} \in I$, otherwise not nilpotent in general. The ideal $R_{n}$ Mat $_{n}(I) R_{n}$ of $R_{n}$ is proper and nilpotent. Hence we conclude that $R_{n}$ is not quasi-IFP.

Now we give a list of rings which coincide on the condition of von Neumann regularity. Here P stands for some property, for instance, property for being reduced, etc.

Lemma 2: Let $A$ be a von Neumann regular ring. Then the following are equivalent.

(P1) $A$ is reduced;

(P2) $A$ is left (or right) duo;

(P3) $A$ is abelian;

(P4) $A$ is 2-primal;

(P5) $A$ is symmetric;

(P6) $A$ is NI;

(P7) $A$ is $N C I$;

(P8) $A$ is IFP;

(P9) $A$ is quasi-IFP;

(P10) $A$ is near-IFP;

(P11) $A$ is a subdirect product of division ring;

(P12) $A$ is Armendariz;

(P13) $A$ is weak Armendariz;

(P14) If $a, a^{\prime}, a^{\prime \prime} \in A$, such that $a a^{\prime \prime}=0=a^{\prime 2}$ with $n \geq 1$, then $a a^{\prime} a^{\prime \prime}=0$.

(P15) If $a, a^{\prime}, a^{\prime \prime} \in A$, such that $a a^{\prime \prime}=0=a^{\prime 2}$, then $a a^{\prime} a^{\prime \prime}=0$.

Proof: The equivalence (P1) $\Leftrightarrow(\mathrm{P} 5)$ is proved in the Theorem above. Equivalences of (P1)-(P4) and (P6) and
(P7) hold in [11; Proposition 1.4]. It is clear by definitions that every reduced ring is IFP, every IFP ring is quasi-IFP and every quasi-IFP ring is near IFP. Thus

$$
(\mathrm{P} 1) \Rightarrow(\mathrm{P} 8) \Rightarrow(\mathrm{P} 9) \Rightarrow(\mathrm{P} 10) \text {. }
$$

Because a von Neumann regular ring is semiprime, by [10; Proposition 1.4], for a semiprime ring a near-IFP ring is reduced, giving the equivalence $(\mathrm{P} 1) \Leftarrow(\mathrm{P} 10)$. Equivalences of (P1)-(P3), (P6), (P8) and (P10) also hold in [10; Proposition 1.6]. The equivalence of a von Neumann regular ring to be reduced, Armendariz, and weak Armendariz is proved in [14; Lemma 2.4]. Finally, the equivalences

$$
\begin{aligned}
& (\mathrm{P} 1) \Leftrightarrow(\mathrm{P} 2) \Leftrightarrow(\mathrm{P} 3) \Leftrightarrow(\mathrm{P} 8) \Leftrightarrow(\mathrm{P} 11) \Leftrightarrow(\mathrm{P} 12) \\
& \Leftrightarrow(\mathrm{P} 13) \Leftrightarrow(\mathrm{P} 14) \Leftrightarrow(\mathrm{P} 15)
\end{aligned}
$$

are established in [14; Lemma 2.4].

Lemma 3 Let $A$ be a semiprime ring of bounded index of nilpotency. Then the following conditions are equivalent:

(P1) $A$ is reduced;

(P4) $A$ is 2-primal;

(P6) $A$ is $N I$;

(P7) $A$ is NCI;

(P8) $A$ is IFP;

(P9) $A$ is quasi-IFP.

Proof: (P1) $\Leftrightarrow(\mathrm{P} 4) \Leftrightarrow(\mathrm{P} 6) \Leftrightarrow$ (P7) hold by [7; Proposition 1.3], (P1) $\Leftrightarrow$ (P6) $\Leftrightarrow$ (P8) $\Leftrightarrow$ (P10) hold by [10; Proposition 1.5] while (P1) $\Leftrightarrow$ (P4) $\Leftrightarrow$ (P6) $\Leftrightarrow(\mathrm{P} 8) \Leftrightarrow(\mathrm{P} 9)$ hold in [11; Proposition 1.6].

The consequences of the Theorem and above lemmas are the following.

Corollary 1: $A C_{k}$-von Neumann regular ring is commutative if any one of the properties (P1)-(P15) of Lemma 2 is satisfied.

Corollary 2: $A C_{k}$-semiprime ring of bounded index of nilpotency is commutative if any one of the properties (P1), (P4), (P6)-(P9) of Lemma 3 is satisfied.

Corollary 3: Let $A$ be a semiprime right Goldie ring and $B$ its classical ring of quotients. Then the ring $B$ satisfies all equivalent conditions from $(P 1)$ to $(P 15)$ of Lemma 2. Moreover, for the ring $A$, the conditions $(P 1)$, (P4), (P8), (P9), (P10), (P12) and (P13) of Lemma 2 are mutually equivalent and are also equivalent to above each of fifteen conditions for the ring $B$.

Proof: Equivalence of (P1) to (P15) is followed from [14; Theorem 2.6] and Lemma 1.2.

If $A$ is near-IFP and semiprime, and if $a$ is nilpotent, then every ideal of $A a A$ is zero. Hence, in particular, $a$ is zero, and $A$ is reduced. So, (P1), (P8)-(P10) are equivalent for the ring $A$. Equivalence of (P1) and (P4) for the ring $A$ is obvious and for the same ring (P12) and (P13) are followed from [14; Theorem 2.6].

Corollary 4: A semiprime right Goldie $C_{k}$-ring is commutative if its classical ring of quotient satisfies any 
one of the properties (P1)-(P15) as listed in Lemma 2.

\section{Acknowledgements}

This project was funded by the Deanship of Scientific Research (DSR), King Abdulaziz University, Jeddah, under grant no. 3-043/429. The authors, therefore, acknowledge with thanks DSR technical and financial support.

\section{REFERENCES}

[1] L. Chuang and J. S. Lin, "On Conjecture of Herstein," Journal of Algebra, Vol. 126, No. 1, 1989, pp. 119-138. doi:10.1016/0021-8693(89)90322-0

[2] A. Klein, I. Nada and H. E. Bell, "Some Commutativity Results for Rings," Bulletin of the Australian Mathematical Society, Vol. 22, No. 2, 1980, pp. 285-289. doi:10.1017/S0004972700006584

[3] J. Lambek, "On the Representation of Modules by Sheaves of Factor Modules," Canadian Mathematical Bulletin, Vol. 14, 1971, pp. 359-368. doi:10.4153/CMB-1971-065-1

[4] J. C. McConnell and R. Robson, "Noncommutative Noetherian Rings,” AMS, 2001.

[5] K. R. Goodearl, "Von Neumann Regular Rings,” Monographs and Studies in Math. 4 Pitman, 1979.

[6] G. Marks, "On 2-Primal Ore Extensions," Communications in Algebra, Vol. 29, No. 5, 2001, pp. 2113-2123. doi:10.1081/AGB-100002173

[7] S. U. Hwang, Y. C. Jeon and K. G. Park, “On NCI
Rings," Bulletin of the Korean Mathematical Society, Vol. 44, No. 2, 2007, pp. 215-223. doi:10.4134/BKMS.2007.44.2.215

[8] G. F. Birkenmeier, H. E. Heatherly and E. K. Lee, “Completely Prime Ideals and Associated Radicals, Ring Theory (Granville, OH 1992)," World Sci. Publ. River H, Edge, 1993, pp. 102-129.

[9] H. E. Bell, "Near-Rings, in Which Every Element Is a Power of Itself," Bulletin of the Australian Mathematical Society, Vol. 2, No. 3, 1970, pp. 363-368. doi:10.1017/S0004972700042052

[10] K. Ham, Y. Jeon, J. Kang, N. Kim, W. Lee, Y. Lee, S. Ryu and H. Yang, "IFP Rings and Near-IFP Rings," Journal of the Korean Mathematical Society, Vol. 45, No. 3, 2008, pp. 727-740. doi:10.4134/JKMS.2008.45.3.727

[11] H. K. Kim, N. K. Kim, M. S. Jeong, Y. Lee, S. J. Ryu and D. E. Yeo, "On Conditions Provided by Nil Radicals," Journal of the Korean Mathematical Society, Vol. 46, 2009, pp. 1027-1040.

[12] M. B. Rege and S. Chhawchharia, "Armendariz Rings," Proceedings of the Japan Academy, Series A, Mathematical Sciences, Vol. 73, No. 1, 1997, pp. 14-17.

[13] T.-K. Lee and T.-L. Wong, "On Armendariz Rings," Houston Journal of Mathematics, Vol. 29, No. 3, 2003, pp. 583-593.

[14] Y. C. Jeon, H. K. Kim, Y. Lee and J. S. Yoon, “On Weak Armendariz Rings," Bulletin of the Mathematical Society, Vol. 46, 2009, pp. 135-136.

[15] G. Marks, “A Taxonomy of 2-Primal Rings,” Journal of Algebra, Vol. 266, No. 2, 2003, pp. 494-520. doi:10.1016/S0021-8693(03)00301-6 